\title{
Is now the right time to abolish breast cancer screening in Hong Kong?
}

\author{
Lorraine CY Chow *, MB, BS, FRCSEd
}

Private Practice

* Corresponding author: drlorrainechow@gmail.com

Hong Kong Med J 2018;24:216-7

DOI: 10.12809/hkmj185079

In a recent article by the Cancer Expert Working Group on Cancer Prevention and Screening, screening for breast cancer in the general female population was not recommended based on the lack of evidence for its survival benefit and the imminent cause of patient anxiety. The criticisms of implementing a population-based screening programme for breast cancer include overexposure to radiation and increment in the number of invasive investigations and treatments for breast lesions that may never become malignant. Nonetheless it is important to recognise that the primary aim of screening in breast cancer is to facilitate timely detection of early-stage disease, and hence improve survival. In contrast to this notion, several large-scale studies using nationwide data showed that screening could only prevent one to two cancer deaths in every one to 2000 women screened at the expense of $20 \%$ overdiagnosis rates as well as induction of anxiety in every one to 2000 women. ${ }^{2,3}$ Nonetheless a more in-depth analysis would reveal major weaknesses in these studies including their methodology, inclusion criteria for screening, level of expertise in the evaluation of screening results, and the standard of equipment used for screening. For instance, the Canadian National Breast Screening Study was the only randomised controlled trial that did not show any survival benefit for screening, but a $35 \%$ increment in the overdiagnosis of ductal carcinoma in situ. ${ }^{4}$ Nonetheless randomisations were not blinded and with pitfalls. Women with symptomatic palpable breast masses were also recruited into the 'screening' arm, and the quality of mammography was suboptimal and evaluation of mammographic images deficient.

On the contrary, many studies have shown that treatment for smaller tumours without nodal involvement confers better oncological outcomes as well as a better chance of undergoing breast conserving surgery, and fewer postoperative morbidities..$^{5-7}$ This is an important issue that was often not addressed by these large-scale population studies such as the Swiss Medical Board study and the Cochrane review. ${ }^{2,3}$ The primary end-point of these studies was reduced mortality. They paid no regard to the short-term physical and psychological impact of cancer treatment. The current trend in treatment for breast cancer is multifaceted. A smaller tumour size increases the chances of breast conserving surgery with consequently less postoperative morbidity compared with standard mastectomy. ${ }^{8}$ Applying the same principle, the advent of sentinel lymph node mapping in the management of the axillary area implied a substantially reduced need for axillary dissection in early tumours, and hence lower risk of lymphoedema and its associated morbidities. ${ }^{9,10}$ Moreover, advances in imaging techniques may further improve the accuracy of screening. In the Norwegian nationwide study of over 40000 women with breast cancer, screening led to a reduction in mortality by 4.8 deaths per 100000 person-years when compared with the nonscreened group. ${ }^{11}$ Furthermore, screening in the 'modern' era further reduced mortality by 7.2 deaths per 100000 person-years compared with screening in the 'historical' era implying that changes to breast cancer awareness, advances in imaging techniques, and improved treatments in recent years could all contribute to the survival benefit of a screening programme. Such finding was also in line with the evidence provided by the National Health Service Screening Programme in which there was a steady decline in mortality for women with breast cancer aged between 50 and 79 years as the screening programme evolved over a 10-year period from 1990 to $2000 .^{12}$ In Hong Kong, there has been a steady increment in the number of new cases of breast cancer over the last three decades. According to the Hong Kong Cancer Registry, breast cancer is now the most common female cancer with over 3500 new cases diagnosed annually. ${ }^{13}$ Public awareness of breast cancer has substantially improved in recent years following promotion by local media and other non-profitable organisations such as the Hong Kong Breast Cancer Foundation, Well Women Clinic of the Tung Wah Group of Hospitals, and the Family Planning Association. In fact, breast screening in our local population has been shown to be feasible and well accepted. ${ }^{14}$ Advanced imaging technology such as three-dimensional mammography has been introduced as an alternative efficient assessment tool for screening as well as multidisciplinary management of breast cancer in clinical practice. It may be premature to conclude that screening for the general female population in Hong Kong is of little clinical value. 


\section{Declaration}

The author has no conflicts of interest to disclose.

\section{References}

1. Cancer Expert Working Group on Cancer Prevention and Screening. Recommendations on prevention and screening for breast cancer in Hong Kong. Hong Kong Med J 2018;24:298-306.

2. Gøtzsche PC, Jørgensen KJ. Screening for breast cancer with mammography. Cochrane Database Syst Rev 2013;(6):CD001877.

3. Biller-Andorno N, Jüni P. Abolishing mammography screening programs? A view from the Swiss Medical Board. N Engl J Med 2014;370:1965-7.

4. Miller AB, Baines CJ, To T, Wall C. Canadian National Breast Screening Study: 2. Breast cancer detection and death rates among women aged 50 to 59 years. CMAJ 1992;147:1477-88.

5. Cossetti RJ, Tyldesley SK, Speers CH, Zheng Y, Gelmon KA. Comparison of breast cancer recurrence and outcome patterns between patients treated from 1986 to 1992 and from 2004 to 2008. J Clin Oncol 2015;33:65-73.

6. Metzger-Filho O, Sun Z, Viale G, et al. Patterns of recurrence and outcome according to breast cancer subtypes in lymph node-negative disease: results from international breast cancer study group trials VIII and IX. J
Clin Oncol 2013;31:3083-90.

7. Jacobson JA, Danforth DN, Cowan KH, et al. Ten-year results of a comparison of conservation with mastectomy in the treatment of stage I and II breast cancer. $\mathrm{N}$ Engl J Med 1995;332:907-11.

8. Eby PR. Evidence to support screening women annually. Radiol Clin North Am 2017;55:441-56.

9. Veronesi U, Paganelli G, Viale G, et al. A randomized comparison of sentinel-node biopsy with routine axillary dissection in breast cancer. N Engl J Med 2003;349:54653.

10. Bromham N, Schmidt-Hansen M, Astin M, Hasler E, Reed MW. Axillary treatment for operable primary breast cancer. Cochrane Database Syst Rev 2017;(1):CD004561.

11. Kalager M, Zelen M, Langmark F, Adami HO. Effect of screening mammography on breast-cancer mortality in Norway. N Engl J Med 2010;363:1203-10.

12. Blanks RG, Moss SM, McGahan CE, Quinn MJ, Babb PJ. Effect of NHS breast screening programme on mortality from breast cancer in England and Wales, 1990-8: comparison of observed with predicted mortality. BMJ 2000;321:665-9.

13. Hong Kong Cancer Registry. Available from: http://www3. ha.org.hk/cancereg/. Accessed Jun 2018.

14. Kwong A, Cheung PS, Wong AY, et al. The acceptance and feasibility of breast cancer screening in the East. Breast 2008;17:42-50. 